Pacific Journal of Mathematics

AN OBSTRUCTION TO FINDING A FIXED POINT FREE MAP 


\title{
AN OBSTRUCTION TO FINDING A FIXED POINT FREE MAP ON A MANIFOLD
}

\author{
Max K. Agoston
}

The problem of whether a manifold $M$ admits a fixed point free map is an old one. One well known result is that if the Euler characteristic $\chi(M)=0$, then $M$ has such a map. In the case where $M$ is a closed differentiable manifold this follows easily from the fact that $\chi(M)=0$ if and only if the tangent bundle of $M$ admits a nonzero cross-section (see Hopf [4]). But $\chi\left(S^{2 n}\right)=2$, and $S^{2 n}$ certainly admits a fixed point free map, namely, the antipodal map. Therefore, the vanishing of the Euler characteristic of a manifold is only a sufficient, though hardly a necessary, condition for the manifold to have a fixed point free map. In the search for other invariants it is natural to generalize somewhat and state the problem in terms finding coincidence free maps.

The object of this paper is to give an elementary proof of the fact that, given a continuous map $f:\left(W^{n}, \delta W^{n}\right) \rightarrow\left(M^{n}\right.$, $\left.\partial M^{n}\right)$ between oriented $C^{\infty}$-manifolds, there is a well defind obstruction $o(f)$ to finding a special sort of map $F: M \rightarrow M$ with the property that $F(x) \neq f(x)$ for all $x \in W$. This is the content of Theorem 1 in $\S 2$. $F$ will not necessarily be homotopic to $f$, but then this is something that should not be required in view of the fact that the antipodal map on $S^{2 n}$ is not homotopic to the identity map either. In Theorem 2 we prove that $o$ (identity) behaves naturally with respect to tangential maps.

The author would like to thank the referee for bringing the work of F. B. Fuller ([2] and [3]) and E. Fadell ([1]) to his attention. In $\S 3$ we discuss the relationship between this paper and their work and how Theorem 1 might be generalized to the case $f: K^{n} \rightarrow M^{n}$, where $K$ is an $n$-complex and $M$ is a 1-connected closed manifold. One of the main differences between our approach and that of Fuller, Fadell, and others who have worked on the question of coincidences of maps is that they have allowed themselves to make changes only by a homotopy. They obtain fairly complete results with that restriction in terms of Lefschetz numbers (see [1]). On the other hand, we partially free ourselves from this requirement (in the sense that our maps will be homotopic only on the $(n-1)$-skeleton in general), so that there are more possibilities for $F$.

Finally, we have restricted ourselves to differentiable manifolds because all the constructions and proofs, which are quite simple from 
a geometric point of view, follow from a few elementary facts about $C^{\infty}$-manifolds. In particular, it is because we have an exponential map from the tangent bundle of these manifolds to the manifold that allows us to use a nonzero vector field over the $(n-1)$-skeleton (which always exists) to homotope any given map to another which is coincidence free with the first on the $(n-1)$-skeleton. Thus, the whole problem reduces to being able to make maps on a disk coincidence free in a certain way, which also is not hard. Most probably, the results themselves are true for topological manifolds, but the proofs would be more technical.

1. The definition of $o(f, g, \sigma)$. Throughout this paper all manifolds will be compact, connected, oriented and $C^{\infty}$. If $M$ is a manifold, then $T M_{x}$ will denote the tangent space of $M$ at $x$ and $\exp _{x}: T M_{x} \rightarrow$ $M$ will be the standard exponential map. (NOTE. $\exp _{x}$ will not be defined everywhere if $M$ has a boundary.) We assume that a base point $p$ has been chosen in the interior of $M$, int $(M)$. Set $M_{0}=$ $M-p$. Furthermore, the metric on $M$ will be induced from a suitable Riemannian metric on its tangent bundle. A good reference for most of the facts we need to know about vector fields is Milnor [8].

Consider a continuous map $f:\left(W^{n}, \partial W^{n}\right) \rightarrow\left(M^{n}, \partial M^{n}\right), n \geqq 1$.

Let $D_{1}$ be the closed $n$-disk in int $(M)$ centered at $p$ and of radius $\delta>0$. Choose a differentiable map $g:(W, \partial W) \rightarrow(M, \partial M)$ which is an $\varepsilon$-approximation to $f$ for some $\varepsilon>0$, i.e., dist $(f(x), g(x))<\varepsilon$ for all $x \in W$. Just how small $\delta$ and $\varepsilon$ have to be will be determined as we go along. That we are able to make the desired choices will be justified by the fact that we can always change the Riemannian metric on $M$. First of all, $\delta$ and $\varepsilon$ must satisfy

(I) $\varepsilon<\operatorname{dist}\left(x, \exp _{x}(v)\right)<\delta / 3$ for each $x \in M$ and each $v \in T M_{x}$ in the domain of $\exp _{x}$ with $\|v\|=1$.

By choosing a small enough $\delta$, we may assume that $g$ is transverse regular on $\partial D_{1}$, and that if $B_{1}, \cdots, B_{k}$ are the components of $g^{-1}\left(D_{1}\right)$ then $B_{i}$ is an $n$-disk in $\operatorname{int}(W)$ and $g \mid B_{i}$ is a diffeomorphism of $B_{i}$ onto $D_{1}$. Let $D$ be the closed $n$-disk centered at $p$ and of radius $\varepsilon / 2$. Set $C_{i}=g^{-1}(D) \cap B_{i}$ and $N=W-\operatorname{int}\left(C_{1} \cup \cdots \cup C_{k}\right)$. Figure 1 below should help in keeping track of the various definitions.

Next, let $\sigma$ be a nondegenerate vector field on $M$ which points inward at all boundary points of $M$ and such that the isolated zeros of $\sigma$ all lie in int $(D)$. Fix distinct $v_{i} \in T M_{p}, 1 \leqq i \leqq 2(k-1)$, with $\left\|v_{i}\right\|=1$ and let $S_{i}=\left\{\exp _{p}\left(t v_{i}\right) \mid \varepsilon / 2 \leqq t \leqq \delta\right\}$. We require further that $\sigma(x)$ is transverse to the tangent vector of $S_{i}$ at every $x \in S_{i}$ and "pointing away from $p . "$ Define $h: N \rightarrow M$ by

$$
h(x)=\exp _{g(x)}(\sigma(g(x)) /\|\sigma(g(x))\|) .
$$




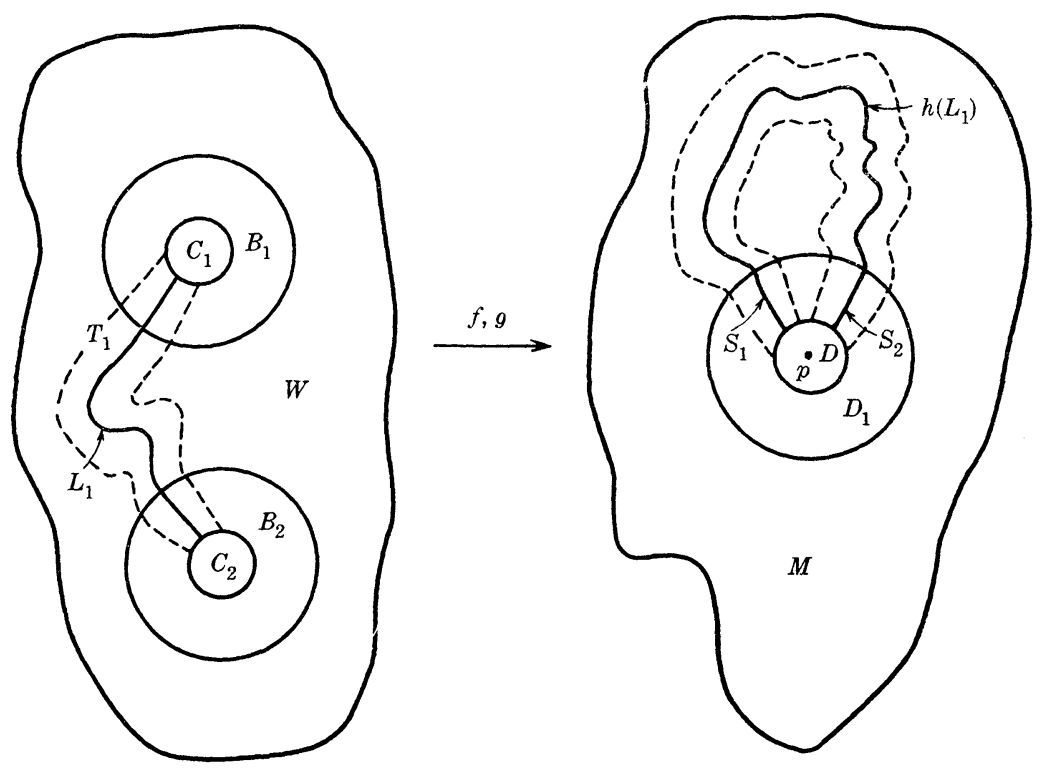

FIGURE 1

With the right Riemannian metric on $M, h$ will be well defined since $\sigma$ points inward on $\partial M$. Clearly, $h$ is homotopic to $f \mid N$. It also follows from the definition of $\sigma$ on the $S_{i}$ and (I) that

(II) $h(x) \neq f(x)$, for $x \in N$, and $h\left((\partial N-\partial W) \cup g^{-1}\left(S_{1}\right) \cup \cdots \cup\right.$ $\left.g^{-1}\left(S_{2(k-1)}\right)\right) \subseteq M-\operatorname{int}(D)$; in fact, $h(\partial N-\partial W) \subseteq D_{1}-\operatorname{int}(D)$.

Case 1. Assume that $n \geqq 3$. Then we can connect the $C_{i}$ with small tubes. Let $\varphi_{i}:[0,1] \rightarrow N$ be disjoint imbeddings which meet $\partial N$ transversally and such that $\varphi_{i}(0) \in \partial C_{i}, \varphi_{i}(1) \in \partial C_{i+1}, g\left(\varphi_{i}([0,1]) \cap B_{i}\right)=S_{2 i-1}$, and $g\left(\varphi_{i}([0,1]) \cap B_{i+1}\right)=S_{2 i}$. Set $L_{i}=\varphi_{i}([0,1])$ and let $T_{i}$ be a tubular neighborhood of $L_{i}$ in $N$. We may suppose that $T_{i} \cap T_{j}=\varnothing=g\left(T_{i}\right) \cap$ $g\left(T_{j}\right)$, for $i \neq j$. Define $B=g^{-1}(D) \cup T_{1} \cup \cdots \cup T_{k-1}$ and $Y=\partial B \cup$ $T_{1} \cup \cdots \cup T_{k-1} \cdot B$ is a closed $n$-disk. (I) and (II) imply that if we choose the $T_{i}$ small enough, then

$$
h(Y) \subseteq M-\operatorname{int}(D) \text {. }
$$

Assume that this is done. Therefore, the problem of extending $h$ to a map $F: W \rightarrow M$ with $F(x) \neq f(x)$ for all $x \in W$ reduces to finding an extension $h_{0}: B \rightarrow M-\operatorname{int}(D)$ of $h \mid \partial B$ with $h_{0}(x) \neq f(x)$ for $x \in B$.

Let $q \in \partial D$ and let $G=\pi_{n-1}\left(M_{0}, q\right)$. The orientation of $W$ induces an orientation on $\partial C_{i}$; and therefore, in the notation of $\mathrm{Hu}$ [5] we get well defined obstructions $\gamma_{i}=\gamma^{n}\left(h \mid \partial C_{i}\right) \in H^{n}\left(C_{i}, \partial C_{i} ; G\right)$ with the property that $\gamma_{i}=0$ if and only if $h \mid \partial C_{i}$ extends to a map $h_{i}: C_{i} \rightarrow M-\operatorname{int}(D)$, since $M-\operatorname{int}(D)$ is a deformation retract of $M_{0}$.

Next, let $W_{0}=W-z$, where $g(z)=p$, and let $Q_{i}=B-\operatorname{int} C_{i}$. Consider 


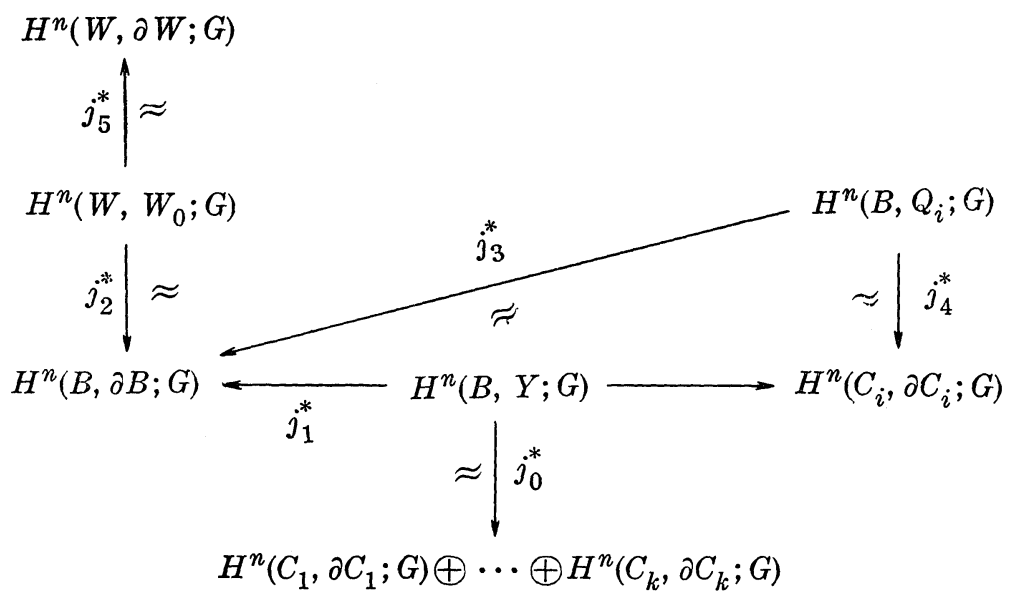

where $j_{i}^{*}, i=0,1, \cdots, 5$, are induced from the natural inclusion maps. Define $\lambda_{i} \in H^{n}(W, \partial W ; G)$ by the equation

$$
j_{4}^{*} j_{3}^{*-1} j_{2}^{*} j_{5}^{*-1}\left(\lambda_{i}\right)=\gamma_{i} .
$$

Definition. $o(f, g, \sigma)=\lambda_{1}+\cdots+\lambda_{k} \in H^{n}(W, \partial W ; G)$.

Let $a_{i} \in \pi_{1}\left(M_{0}, q\right)$ be the elements determined by $g\left(L_{i}\right)$. Recall that $\pi_{1}\left(M_{0}, q\right)$ acts on $G$. This action induces an action of $\pi_{1}\left(M_{0}, q\right)$ on $H^{n}(B, \partial B ; G)$. Then it is easy to check from the definitions that the obstruction $\gamma^{n}(h \mid \partial B) \in H^{n}(B, \partial B ; G)$ satisfies $\gamma^{n}(h \mid \partial B)=j_{1}^{*}\left(j_{0}^{*-1}\left(\gamma_{k}\right)\right)+$ $a_{k-1} \cdot j_{1}^{*}\left(j_{0}^{*-1}\left(\gamma_{k-1}\right)\right)+\cdots+a_{1} \cdot j_{1}^{*}\left(j_{0}^{*-1}\left(\gamma_{1}\right)\right)$. It follows that $j_{2}^{*} j_{5}^{*-1}(o(f, g$, $\sigma))=\gamma^{n}(h \mid \partial B)$ if the $a_{i}$ act trivially on $H^{n}(B, \partial B ; G)$.

Definition. $f$ is said to satisfy $\left(^{*}\right)$ if the above construction can be performed in such a way that $a_{i} \cdot j_{1}^{*}\left(j_{0}^{*-1}\left(\gamma_{i}\right)\right)=j_{1}^{*}\left(j_{0}^{*-1}\left(\gamma_{i}\right)\right)$.

Lemma 1. Let $n \geqq 3$. $f$ will satisfy $\left(^{*}\right)$ in any one of the following situations:

(a) $M_{0}$ is $(n-1)$-simple; in particular, if $M$ is 1-connected.

(b) $f_{\sharp}: \pi_{1}(W, z) \rightarrow \pi_{1}(M, p)$ is onto, or the $a_{i}$ above are zero.

(c) For some $g, k=1$ above. For example, this is so if $f=$ identity.

Proof. If $M$ is 1 -connected, then $M_{0}$ is 1 -connected because $n \geqq$ 3. Therefore, (a) is obvious. (c) and the second part of (b) are also obvious. The first part of (b) follows from the fact that in that case we can always choose our paths $L_{i}$ so that $a_{i}=0$. We need $n \geqq 3$ here in order to be able to realize homotopy classes of paths by imbeddings.

Let us summarize our discussion in a lemma. 
Lemma 2. Let $n \geqq 3$. Suppose $f$ satisfies $(*)$ and we have performed the constructions above with respect to a vector field $\sigma$ and approximation $g$. Then there is a well defined obstruction $o(f, g, \sigma) \in$ $H^{n}(W, \partial W ; G)$ with the property that $o(f, g, \sigma)=0$ if and only if there exists a map $h_{1}: W \rightarrow M$ such that

(a) $h_{1} \mid W-\operatorname{int}(B)=h$, and

(b) $h_{1}(B) \subseteq M-\operatorname{int}(D)$.

By construction $h_{1}$ will also have the property that $h_{1}(x) \neq f(x)$ for $x \notin T_{1} \cup \cdots \cup T_{k-1}$. We would like to have $h_{1}$ differ from $f$ on the $T_{i}$ also, but we can arrange this if we are a little more careful about how we define $h$ and $h_{1}$. (NOTE. The $h_{1}$ we get may not agree with $h$ on $T_{i}-\left(\partial B \cap T_{i}\right)$.)

Since $n \geqq 3$, we may assume that $g \mid L_{i}$ are disjoint imbeddings. Furthermore, we can also require that $\sigma(x)$ is transverse to the tangent vector of $g\left(L_{i}\right)$ for every $x \in g\left(L_{i}\right)$ and "pointing away from $p$." Then by changing the Riemannian metric on $M$ and making the $T_{i}$ still smaller, if necessary, we can assume that $h\left(T_{i}\right) \cap f\left(T_{j}\right)=\varnothing$ for all $i$ and $j$. Therefore, $h_{1}\left(\partial T_{i}\right) \cap f\left(T_{j}\right)=\varnothing$. Now homotope $h_{1}\left(T_{i}\right)$ into a neighborhood of $h_{1}(\partial B)$ using $h_{1}(B)$ and keeping $h_{1} \mid \partial B$ fixed. This can be done simultaneously for all $i$. In effect, we push $h_{1}\left(T_{k-1} \cap \partial C_{k}\right)$ through $h_{1}\left(B-C_{k}\right)$ towards $h_{1}\left(\partial B-\partial C_{k}\right)$. We can do this in such a way so that now $h_{1}(x) \neq f(x)$ for $x \in T_{1} \cup \cdots \cup T_{k-1}$. But then $h_{1}(x) \neq f(x)$ for $x \in W$, since we have not disturbed anything on $M-$ $\operatorname{int}(B)$.

We have therefore proved the following:

LEMMA 3. Let $n \geqq 3$ and suppose $f$ satisfies $\left({ }^{*}\right)$. Let $\sigma$ be $a$ vector field on $M$ and $g$ a differentiable approximation to $f$ as described above. Then there is a well defined obstruction $o(f, g, \sigma) \in$ $H^{n}\left(W, \partial W ; \pi_{n-1}\left(M_{0}, q\right)\right)$ with the property that $o(f, g, \sigma)=0$ if and only if there exists a map $F: W \rightarrow M$ such that

(a) $F(x) \neq f(x)$ for all $x \in W$, and

(b) $F(x)=h(x)$ for $x$ outside some open n-disk in $W$, where $h$ is obtained from $\sigma$ and $g$ as above.

Note. $o(f, g, \sigma)$ actually depends also on $p, q$, and the orientations, but the vanishing of $o(f, g, \sigma)$ is independent of this data. Also, since $H^{n}\left(W, \partial W ; \pi_{n-1}\left(M_{0}, q\right)\right) \approx \pi_{n-1}\left(M_{0}, q\right)$, we can think of $o(f$, $g, \sigma)$ as bing an element of $\pi_{n-1}\left(M_{0}, q\right)$.

Definition. Any map $F$ which satisfies the conclusion of Lemma 3 with respect to some $\sigma$ will be called a $\sigma$-map for $f$ to indicate its 
intimate relation to $\sigma$, namely (b).

Case 2. Assume that $n \leqq 2$. In certain cases we can still define an obstruction $o(f, g, \sigma) \in H^{n}\left(W, \partial W ; \pi_{n-1}\left(M_{0}, q\right)\right)$ having the properties described in Lemma 3. $\pi_{n-1}\left(M_{0}, q\right)$ may not be abelian when $n=2$. In that case we identify that cohomology group with $\pi_{n-1}\left(M_{0}, q\right)$. Nevertheless one can manipulate the group formally just like other cohomology groups.

(a) Suppose $M^{2}$ is arbitrary and $f$ is the identity map. If we work directly with $\pi_{1}\left(M_{0}, q\right)$ rather than cohomology groups, then $o$ (identity, identity, $\sigma$ ) can be defined just like in Case 1, because the only difficulties that were encountered there were due to the fact that $k$ could be larger than 1 . ( $k$ was the number of components of $g^{-1}(D)$ for some $n$-disk $D$.)

(b) Suppose $f$ is arbitrary and $M=S^{n}$ or $D^{n}$. If $M=S^{n}$, then $M_{0}$ is contractible and it is easy to show that there is no obstruction at all to getting a $\sigma$-map $F: W \rightarrow S^{n}$ with $F(x) \neq f(x)$ for all $x \in W$ and all vector fields $\sigma$. If $M=D^{n}$, then there is again no difficulty in using the procedure of Case 1 to define an obstruction $o(f, g, \sigma) \in$ $\pi_{n-1}\left(D^{n}-p\right) \approx Z$ or $Z_{2}$ with the desired properties.

Definition. $f$ is said to satisfy $\left({ }^{* *}\right)$ if one of the following holds:

(a) $n \geqq 3$ and $f$ satisfies (*).

(b) $n=2$ and $f$ is the identity.

(c) $M^{n}=S^{n}$ or $D^{n}$.

2. The obstruction $o(f)$. Suppose we are given a continuous map $f:\left(W^{n}, \partial W^{n}\right) \rightarrow\left(M^{n}, \partial M^{n}\right)$ so that $f$ satisfies $\left(^{* *}\right)$. Then we defined in $\S 1$ an obstruction $o(f, g, \sigma) \in H^{n}\left(W, \partial W ; \pi_{n-1}\left(M_{0}, q\right)\right)$ to finding a $\sigma$-map $F: W \rightarrow M$ with $F(x) \neq f(x)$ for all $x \in W$. Now we would like to show that in fact $o(f, g, \sigma)$ does not depend on $\sigma$ or $g$. We retain the notation developed in $\S 1$.

Assume that $n \geqq 2$. Observe that $H^{n}\left(W, \partial W ; \pi_{n-1}\left(D_{1}-p, q\right)\right) \approx$ $\pi_{n \rightarrow 1}\left(D_{1}-p, q\right)=Z$. Let $\eta=$ degree $\left(h \mid \partial C_{1}\right)+\cdots+$ degree $\left(h \mid \partial C_{k}\right) \in$ $H^{n}\left(W, \partial W ; \pi_{n-1}\left(D_{1}-p, q\right)\right)$. (NOTE. degree $\left(h \mid \partial C_{i}\right)=\gamma^{n}\left(h \mid \partial C_{i}\right)$, which is the obstruction to extending $h \mid \partial C_{i}$ to a map from $C_{i} \rightarrow D_{1}-\operatorname{int}(D)$.) It follows from the definitions that if

$$
\begin{aligned}
& \alpha: H^{n}(W, \partial W ; Z)=H^{n}\left(W, \partial W ; \pi_{n-1}\left(D_{1}-p, q\right)\right) \\
& \rightarrow H^{n}\left(W, \partial W ; \pi_{n-1}\left(M_{0}, q\right)\right)
\end{aligned}
$$

is induced by the coefficient homomorphism $\pi_{n-1}\left(D_{1}-p, q\right) \rightarrow \pi_{n-1}\left(M_{0}\right.$, $q)$, then $\alpha(\eta)=o(f, g, \sigma)$. Let $\chi(M)$ denote the Euler characteristic of $M$ and set $d=$ degree $(f)=\operatorname{degree}\left(f \mid B:(B, \partial B) \rightarrow\left(M, M_{0}\right)\right)$. Then 
it is known that $\eta=d \cdot($ index of $\sigma)=d \cdot \chi(M)$, where the index of $\sigma$ is the sum of the indices at the zeros of $\sigma$. (See Milnor [8]; in particular, the Poincaré-Hopf theorem.) This shows that $\eta$, and hence $o(f, g, \sigma)$, is independent of $\sigma$ and $g$.

If $n=1$, then $M=S^{1}$ or $D^{1}$ and it is trivial to see that $o(f, g, \sigma)$ is again independent of $\sigma$ and $g$.

Definition. $o(f) \equiv o(f, g, \sigma)$ for some $\sigma$ and $g$. Let $o(M) \equiv$ $o$ (identity: $M \rightarrow M$ ).

We can now state the first theorem of this paper.

THEOREM 1. Suppose that $f:\left(W^{n}, \partial W^{n}\right) \rightarrow\left(M^{n}, \partial M^{n}\right)$ is a continuous map and that $f$ satisfies (**). Then there is a well defined obstruction $o(f) \in H^{n}\left(W, \partial W ; \pi_{n-1}\left(M_{0}, q\right)\right)$ with the following properties:

(a) $o(f)=0$ if and only if there is a vector field $\sigma$ on $M$ and a $\sigma$-map $F: W \rightarrow M$ with $F(x) \neq f(x)$ for all $x \in W$.

(b) $\alpha($ degree $(f) \cdot \chi(M))=o(f)$.

Proof. Theorem 1 is a consequence of Lemma 3 and the remarks made at the beginning of this section.

Observe that although $F \mid W_{0}$ is homotopic to $f \mid W_{0}, F$ need not be homotopic to $f$. For example, if $W=M=S^{n}$ and $f=$ identity, then $F$ is homotopic to the antipodal map and therefore has degree $(-1)^{n+1}$.

CoRollary 1. Let $f:(W, \partial W) \rightarrow(M, \partial M)$. Then there is a map $F: W \rightarrow M$ such that $F(x) \neq f(x)$ for $x \in W$ if any one of the following conditions is satisfied:

(a) $\chi(M)=0$.

(b) $\pi_{n-1}\left(M_{0}, q\right)=0$.

(c) degree $(f)=0$.

In particular, every homotopy sphere admits a fixed point free map.

Proof. If $f$ satisfies $\left({ }^{* *}\right)$, then the corollary follows from Theorem 1. If $f$ does not satisfy $\left(^{* *}\right)$, then one has to go back and look at the various constructions in the proof of Theorem 1 to see that the corollary is still true.

Note. (a) and (c) in the corollary follow also from the work of [1], and [3] in the case where $M^{n}$ is closed, 1-connected, and $n \geqq 3$.

The condition $\pi_{n-1}\left(M_{0}, q\right)=0$ is of course very strong. Unfortunately, the map $\alpha$ above is often one-to-one so that $o(M)$ gives us no more information than $\chi(M)$ in that case. However, the next corollary 
is useful for certain spherical manifolds. (See [6] for a definition of "spherical.")

CoRollary 2. Let $M^{n}$ be a closed 1-connected manifold and $f: M \rightarrow$ $M$. Suppose that all the nonzero elements of $\pi_{n-1}\left(M_{0}, q\right)$ have order 2. Then there is a map $F: M \rightarrow M$ such that $F(x) \neq f(x)$ for $x \in M$. In particular, $M$ admits a fixed point free map.

Proof. The conditions on $M$ imply that $M$ is spherical and that $\chi(M)=2$ or 0 . The case $\chi(M)=0$ is clear. If $\chi(M)=2$, then $o(f)=$ $\alpha($ degree $(f) \cdot 2)=0$, and we can apply Theorem 1 .

Next, let $f:\left(M^{n}, \partial M^{n}\right) \rightarrow\left(W^{n}, \partial W^{n}\right)$ be a continuous map which preserves the tangent bundles. It is well known that the Euler characteristic behaves naturally with respect to such maps, i.e., $\chi(M)=$ degree $(f) \cdot \chi(W)$. We want to show now that the obstructions defined in this paper satisfy an analogous formula.

Approximate $f$ by a differerentiable map $g:(M, \partial M) \rightarrow(W, \partial W)$. If $\sigma$ is a vector field on $W$, then $g$ will induce a vector field $g^{*} \sigma$ on $M$. We can also find a closed $n$-disk $D \subseteq \operatorname{int} W$ so that $g^{-1}(D)=$ $C_{1} \cup \cdots \cup C_{k}$, where $C_{i}$ is a closed $n$-disk in the interior of $M$ and $g \mid C_{i}: C_{i} \rightarrow D$ is a diffeomorphism. Assume that the zeros of $\sigma$ are contained in int $(D)$. Then all the zeros of $g^{*} \sigma$ will lie in $g^{-1}(D)$. In fact, for each zero of $\sigma$, there will be $k$ zeros of $g^{*} \sigma$ with the same index.

Let $p$ and $z$ be the base points of $M$ and $W$, respectively, and let $n \geqq 2$. By connecting the $C_{i}$ with little tubes we can find a closed $n$-disk $B$ such that $C_{1} \cup \cdots \cup C_{k} \subseteq B \subseteq$ int $M$ and $p \in B$. If we assume that $M_{0}$ and $W_{0}$ are $(n-1)$-simple, then we can ignore base points. Therefore, $g$, and hence $f$, induces a well defined homomorphism $\beta_{f}$ : $\pi_{n-1} M_{0} \rightarrow \pi_{n-1} W_{0}$ because $M-\operatorname{int}(B)$ is deformation retract of $M_{0}$. If we now recall our definitions of $o(M)$ and $o(W)$, then the next theorem follows easily:

THEOREM 2. Let $f:\left(M^{n}, \partial M^{n}\right) \rightarrow\left(W^{n}, \partial W^{n}\right), n \geqq 2$, be a continuous tangential map. Assume that $M_{0}$ and $W_{0}$ are $(n-1)$-simple. Then $\beta_{f}(o(M))=$ degree $(f) \cdot o(W)$, where $\beta_{f}: \pi_{n-1} M_{0} \rightarrow \pi_{n-1} W_{0}$ is the map induced by $f$ as above.

Theorem 2 enables us to compute $o(W)$ once we know $o(M)$ and we have a tangential map of degree 1.

CoRollary 3. If $M_{0}$ is $(n-1)$-simple, then $o(M)$ is an invariant of the tangential homotopy type of $(M, \partial M)$. (Actually, all we need is a tangential homotopy equivalence $f: M \rightarrow W$ such that $f(\partial M) \cong \partial W$ 
and $\operatorname{deg} f=1$.)

3. Relationships to other obstructions. In this section we would like to discuss the relationship between the obstruction $o(f)$ and the ones defined in [1] and [3]. We shall indicate how one might extend Theorem 1 and then make a concluding remark.

Suppose that $M^{n}, n \geqq 3$, is a 1-connected closed manifold and $f: M \rightarrow M$. It follows from [1] and [3] that $\eta=\operatorname{degree~}(f) \cdot \chi(M) \in$ $H^{n}\left(M ; \pi_{n-1}\left(D_{1}-p, q\right)\right)=H^{n}(M ; Z)=Z$ is the obstruction to finding a map $F: M \rightarrow M$ such that $F$ is homotopic to $f$ and $F(x) \neq f(x)$ for all $x \in M$. This and Theorem $1(\mathrm{~b})$ shows that $o(f)$ is a weaker obstruction than that in [1] and [3], since the latter maps onto the former under $\alpha$. Note that the $F$ in Theorem 1(a) will be homotopic to $f$ if and only if degree $(f) \cdot \chi(M)=0$.

Next, if we drop our hypothesis of differentiability and use the techniques of [1], [2], and [3] instead, it is most probable that the following extension of Theorem 1 is true:

CONJECTURE. Let $M^{n}, n \geqq 3$, be a 1-connected closed topological manifold. Suppose that $K$ is a finite $n$-complex and that $f: K \rightarrow M^{n}$ is a continuous map. Then there is a well defined obstruction $o(f) \in$ $H^{n}\left(K ; \pi_{n-1}\left(M_{0}, q\right)\right)$ such that $o(f)=0$ if and only if there exists a map $F: K \rightarrow M$ with $F(x) \neq f(x)$ for all $x \in K$ and $F$ homotopic to $f$ on the $(n-1)$-skeleton of $K$.

Finally, this paper suggests that the next step in the program for determining when a manifold admits a fixed point free map is to allow maps to have even more freedom and require that they be homotopic (via a vector field) only over the $(n-2)$-skeleton, then only over the $(n-3)$-skeleton, and so on. This process may lead to a nice well defined sequence of obstructions which might be computable in certain cases.

\section{REFERENCES}

1. E. Fadell, On a coincidence theorem of F. B. Fuller, Pacific J. Math., 15 (1965), 824-834.

2. F. B. Fuller, The homotopy theory of coincidences, Princeton thesis, 1951.

3. The homotopy theory of coincidences, Ann. of Math., 59 (1954), 219-226.

4. H. Hopf, Vektorfelder in n-dimensionaler Mannigfaltigkeiten, Math. Ann., 96 (1926), 225-250.

5. Sze-Tsen Hu, Homotopy theory, Academic Press, New York, 1959.

6. K. W. Kwun and F. Raymond, Spherical manifolds, Duke Math. J., 34 (1967), 397401.

7. J. Milnor, Morse theory, Princeton University Press, 1963.

8. Topology from the differentiable viewpoint, The University Press of Virginia, Charlottesville, 1965.

Received May 5, 1970 and in revised form June 7, 1972. This work was partially supported by NFS Grant No. GP-29552. 



\section{PACIFIC JOURNAL OF MATHEMATICS}

\section{EDITORS}

\author{
H. SAMELSON \\ Stanford University \\ Stanford, California 94305 \\ C. R. HOBBY \\ University of Washington \\ Seattle, Washington 98105
}

\section{J. DugundjI}

Department of Mathematics University of Southern California Los Angeles, California 90007

\section{RICHARD ARENS}

University of California

Los Angeles, California 90024

\section{ASSOCIATE EDITORS}
E. F. BECKENBACH
B. H. NeumanN
F. WOLF
K. YosHIDA

\section{SUPPORTING INSTITUTIONS}

\author{
UNIVERSITY OF BRITISH COLUMBIA \\ CALIFORNIA INSTITUTE OF TECHNOLOGY \\ UNIVERSITY OF CALIFORNIA \\ MONTANA STATE UNIVERSITY \\ UNIVERSITY OF NEVADA \\ NEW MEXICO STATE UNIVERSITY \\ OREGON STATE UNIVERSITY \\ UNIVERSITY OF OREGON \\ OSAKA UNIVERSITY
}

\author{
UNIVERSITY OF SOUTHERN CALIFORNIA \\ STANFORD UNIVERSITY \\ UNIVERSITY OF TOKYO \\ UNIVERSITY OF UTAH \\ WASHINGTON STATE UNIVERSITY \\ UNIVERSITY OF WASHINGTON \\ ${ }^{*} \stackrel{*}{*}{ }^{*}$
AMERICAN MATHEMATICAL SOCIETY
NAVAL WEAPONS CENTER
}

The Supporting Institutions listed above contribute to the cost of publication of this Journal, but they are not owners or publishers and have no responsibility for its content or policies.

Mathematical papers intended for publication in the Pacific Journal of Mathematics should be in typed form or offset-reproduced, (not dittoed), double spaced with large margins. Underline Greek letters in red, German in green, and script in blue. The first paragraph or two must be capable of being used separately as a synopsis of the entire paper. The editorial "we" must not be used in the synopsis, and items of the bibliography should not be cited there unless absolutely necessary, in which case they must be identified by author and Journal, rather than by item number. Manuscripts, in duplicate if possible, may be sent to any one of the four editors. Please classify according to the scheme of Math. Rev. Index to Vol. 39. All other communications to the editors should be addressed to the managing editor, Richard Arens, University of California, Los Angeles, California, 90024.

50 reprints are provided free for each article; additional copies may be obtained at cost in multiples of 50 .

The Pacific Journal of Mathematics is published monthly. Effective with Volume 16 the price per volume (3 numbers) is $\$ 8.00$; single issues, $\$ 3.00$. Special price for current issues to individual faculty members of supporting institutions and to individual members of the American Mathematical Society: $\$ 4.00$ per volume; single issues $\$ 1.50$. Back numbers are available.

Subscriptions, orders for back numbers, and changes of address should be sent to Pacific Journal of Mathematics, 103 Highland Boulevard, Berke'ey, California, 94708.

PUBLISHED BY PACIFIC JOURNAL OF MATHEMATICS, A NON-PROFIT CORPORATION

Printed at Kokusai Bunken Insatsusha (International Academic Printing Co., Ltd.), 270, 3-chome Totsuka-cho, Shinjuku-ku, Tokyo 160, Japan. 


\section{Pacific Journal of Mathematics}

\section{Vol. 43, No. $3 \quad$ May, 1972}

Max K. Agoston, An obstruction to finding a fixed point free map on a manifold.... 543

Nadim A. Assad and William A. Kirk, Fixed point theorems for set-valued mappings

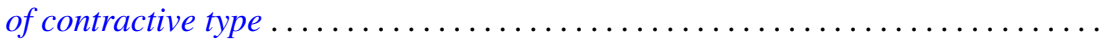

John Winston Bunce, Characterizations of amenable and strongly amenable

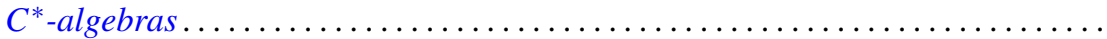

Erik Maurice Ellentuck and Alfred Berry Manaster, The decidability of a class of

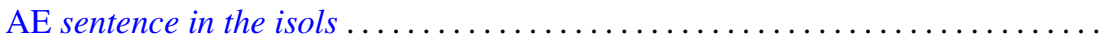

U. Haussmann, The inversion theorem and Plancherel's theorem in a Banach

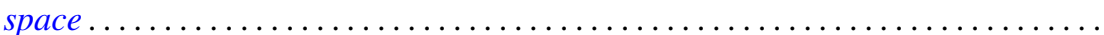

Peter Lawrence Falb and U. Haussmann, Bochner's theorem in infinite dimensions.

Peter Fletcher and William Lindgren, Quasi-uniformities with a transitive base ..... Dennis Garbanati and Robert Charles Thompson, Classes of unimodular abelian

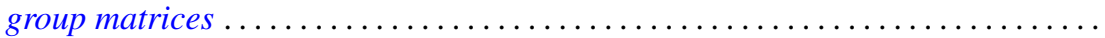

Kenneth Hardy and R. Grant Woods, On c-realcompact spaces and locally bounded normal functions

Manfred Knebusch, Alex I. Rosenberg and Roger P. Ware, Grothendieck and Witt rings of hermitian forms over Dedekind rings .......................

George M. Lewis, Cut loci of points at infinity.

Jerome Irving Malitz and William Nelson Reinhardt, A complete countable $L_{\omega_{1}}^{Q}$ theory with maximal models of many cardinalities . . . . . . . . . . . . . . . . .

Wilfred Dennis Pepe and William P. Ziemer, Slices, multiplicity, and Lebesgue

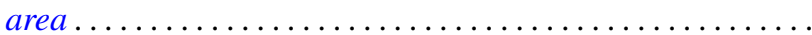

Keith Pierce, Amalgamating abelian ordered groups . .

Stephen James Pride, Residual properties of free groups . . . . . . . . . . . . . 725

Roy Martin Rakestraw, The convex cone of n-monotone functions .

T. Schwartzbauer, Entropy and approximation of measure preserving transformations .

Peter F. Stebe, Invariant functions of an iterative process for maximization of a polynomial...

Kondagunta Sundaresan and Wojbor Woyczynski, L-orthogonally scattered

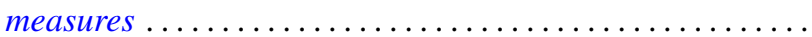

Kyle David Wallace, $C_{\lambda}$-groups and $\lambda$-basic subgroups $\ldots \ldots \ldots$

Barnet Mordecai Weinstock, Approximation by holomorphic functions on certain

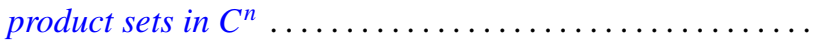

Donald Steven Passman, Corrections to: "Isomorphic groups and group rings”.

Don David Porter, Correction to: "Symplectic bordism, Stiefel-Whitney numbers, and a Novikov resolution"

John Ben Butler, Jr., Correction to: “Almost smooth perturbations of self-adjoint operators".

Constantine G. Lascarides, Correction to: "A study of certain sequence spaces of Maddox and a generalization of a theorem of Iyer" ...... ...

George A. Elliott, Correction to: "An extension of some results of takesaki in the reduction theory of von neumann algebras" ......................... 